Check for updates

Cite this: RSC Adv., 2019, 9, 18377

\title{
Fluorinated graphdiyne as a significantly enhanced fluorescence material $\dagger$
}

\author{
Wenqing Xiao, ${ }^{\text {abc }}$ Huifang Kang, ${ }^{\text {abc }}$ Yuda Lin, ${ }^{\text {abc }}$ Mingxing Liang, ${ }^{\text {abc }}$ Jiaxin Li, ${ }^{\text {abc }}$ \\ Feng Huang, (D) abc Qian Feng, (D) *abc Yongping Zheng ${ }^{\star a b c}$ and Zhigao Huang (D) abc
}

The chemical modification of graphdiyne (GDY) using light elements is a possible route to regulate its unique structure and optoelectronic properties. In this paper it is shown that directly heating a mixture of xenon difluoride and GDY produces partially fluorinated GDY with covalent $\mathrm{C}-\mathrm{F}$ bonding and localized $\mathrm{sp}^{2}$-carbon hybridization because of the breaking of the acetylenic bond. It is seen that the fluorescence of GDY is significantly enhanced because of the fluorine doping. All the fluorinated GDYs with different doping ratios of fluorine exhibit photoluminescence from bright blue to green when the excitation wavelength varies from $260 \mathrm{~nm}$ to $480 \mathrm{~nm}$. In addition, the doped GDY with $15.2 \%$ fluorine doping shows a strong photoluminescence and the quantum efficiency is $3.7 \%$. The enhanced fluorescence is considered to be induced by defect states because of the doping of fluorine, suggesting its potential applications in luminescence devices, such as biological sensing and flexible light-emitting diodes.

Received 25th March 2019 Accepted 14th May 2019

DOI: 10.1039/c9ra02272d

rsc.li/rsc-advances of copper were successfully synthesized in $2010 .^{9-12}$ According to density functional theory (DFT), the direct band gap of original GDY is from $0.44 \mathrm{eV}$ to $1.10 \mathrm{eV}$ with different approximate methods, ${ }^{13}$ which shows that GDY has an advantage of over graphene for optoelectronic applications. ${ }^{14}$ The GDY and related materials were believed to be promising PL materials, which may be used as a highly efficient photocatalyst or for fluorescence sensing, and so on ${ }^{3,15,16}$ Compared with traditional optoelectronic materials, GDY has higher optical responsivity and faster response rate, which is attributed to its better conductivity. ${ }^{16}$ However, there are few studies on the intrinsic fluorescence spectra or fluorescence mechanism of GDY because of its low emission efficiency, and it may still have a far reaching significance worth exploring.

Doping of carbon-based materials using light elements is an efficient method to modify the electric and optoelectronic properties. ${ }^{17,18}$ It was confirmed that the doping of fluorine on the carbon framework of graphene can tune the gap from $0 \mathrm{eV}$ to $\sim 3 \mathrm{eV} .^{6}$ The fluorinated graphene can emit visible PL using irradiation of near-ultraviolet rays. ${ }^{6,7}$ The GDY has one-third of $\mathrm{sp}^{2}$ bonds which is similar to that of graphene. Because of the similar structure of graphene and GDY, research on fluorinated graphdiyne (FGDY) is also underway. It has been reported that FGDY also has excellent properties of energy storage. ${ }^{19}$ It is also shown that using FGDY is more advantageous than using fluorinated graphene in the band gap adjustment of nano optoelectronic materials. ${ }^{20}$ Compared to graphene, GDY has additional sp bonds, which means that the light atoms have more abundant doping sites than graphene, which can cause more interesting PL.
${ }^{a}$ College of Physics and Energy, Fujian Normal University, Fujian Provincial Key Laboratory of Quantum Manipulation and New Energy Materials, Fuzhou, 350117, China. E-mail: fengqian@fjnu.edu.cn; zyp@fjnu.edu.cn; Fax: +86-591-22867577; Tel: +86-591-22867577

${ }^{b}$ Fujian Provincial Collaborative Innovation Center for Optoelectronic Semiconductors and Efficient Devices, Xiamen University, Xiamen, 361005, China

${ }^{c}$ Fujian Provincial Engineering Technology Research Center of Solar Energy Conversion and Energy Storage, Fuzhou 350117, China

$\dagger$ Electronic supplementary information (ESI) available: Schematic illustrations of F doping in GDY: the PL spectra at different excitation wavelengths from $260 \mathrm{~nm}$ to $480 \mathrm{~nm}$ of GDY, FGDY-1 and FGDY-3. See DOI: 10.1039/c9ra02272d 
In the research reported here, a simple method was used for the synthesis of FGDYs with covalent C-F bonding and localized $\mathrm{sp}^{2}$-carbon hybridization. For the pristine GDY which has a residual oxygen content, then the contribution of oxygen should be considered for the FGDYs. The PL properties were then explored, and it is found that with the introduction of fluorine atoms, the fluorescence intensity of FGDYs is greatly enhanced when compared to that of the original GDY. The FGDY with $15.2 \%$ fluorine doping shows a strong blue luminescence and the quantum efficiency is $3.7 \%$. Because of the low phonon energy of fluoride, doping $\mathrm{F}$ atoms makes it possible to reduce or eliminate non-radiative transition sites. The enhanced fluorescence of FGDY suggests it has potential applications in luminescence devices, such as biological sensing and flexible light-emitting diodes.

\section{Experimental}

\subsection{Preparation of GDY}

The GDY was synthesized using a cross-coupling reaction with hexaethynylbenzene as the precursor. ${ }^{9}$ Tetrabutylammonium fluoride was added to the tetrahydrofuran solution of hexakis [(trimethylsilyl)-ethynyl]benzene in an ice bath, then it was stirred until the solution turned purple. The solution was washed with sodium chloride, dried over magnesium sulfate and filtered, and then evaporated under vacuum to synthesize the hexaethynylbenzene monomer. The GDY was grown in the presence of pyridine and copper power using a cross-coupling reaction of the monomer of hexaethynylbenzene for $72 \mathrm{~h}$ at $120{ }^{\circ} \mathrm{C}$ under an argon atmosphere and in dark conditions. After the reaction, the black solution was evaporated at $60{ }^{\circ} \mathrm{C}$ to remove pyridine. The powder obtained was purified and washed sequentially with acetone, hot dimethylformamide and ethanol. Then, the powder was washed several times sequentially with $4 \mathrm{M}$ sodium hydroxide, $6 \mathrm{M}$ hydrochloric acid, ethanol, and deionized water to remove metallic ions and other inorganic salt. After drying, the GDY powder was obtained.

\subsection{Preparation of FGDY}

The FGDY was prepared by directly heating a certain mass ratio of the mixture of GDY and xenon difluoride $\left(\mathrm{XeF}_{2}\right)$ in a Teflon container at $180{ }^{\circ} \mathrm{C}$ for $12 \mathrm{~h}$ under an argon atmosphere. In order to study the effect of fluorination on the fluorescence of the doped samples, the same pristine GDY is used, and the degree of fluorination was varied by using different mass ratios of $\mathrm{XeF}_{2}$ and GDY. The mass ratios of GDY and $\mathrm{XeF}_{2}$ were $1: 2$, $1: 4$, or $1: 8$. The corresponding samples were denoted as FGDY-1, FGDY-2 and FGDY-3, respectively.

\subsection{Characterization}

The morphology of the prepared samples was observed using scanning electron microscopy (JSM-7500F, Jeol, Japan) and transmission electron microscope (TEM, G2 F20, Tecnai, Hillsboro, OR, USA). The chemical composition of the samples was determined using X-ray photoelectron spectroscopy (XPS, ESCALAB 250, VG Systems, USA). The Raman spectra was measured using a micro-Raman system (LabRAM-HR, Horiba, Longjumeau, France). The surface environment of the samples were observed using Kelvin probe atomic force microscopy (AFM, Dimension Icon, Bruker, Germany). The PL excitation and emission spectra were monitored using a fluorescence spectrometer (Fluorolog-3 FL3-22, Horiba Jobin Yvon, France).

\subsection{Measurement of quantum yield}

The QY was calculated using to the following equation:

$$
Q=Q_{\mathrm{r}}\left(I / I_{\mathrm{r}}\right)\left(A_{\mathrm{r}} / A\right)
$$

where $Q$ is the QY of the FGDYs and the standard substance, $I$ is the measured integrated emission intensity, and $A$ is the maximum absorbance value. The subscript ' $r$ ' refers to the standard.

\section{Results and discussion}

Fig. 1a shows the AFM image of FGDY-2. It exhibits a sheet-like structure and its surface was uneven. The TEM image of FGDY-2 (Fig. 1b) further confirmed a more uniform overlapping multilayer structure. It can be seen that it is only the ultrasonic treatment which does not make single layered FGDYs or with a small layer structure. ${ }^{21}$ Fig. $1 \mathrm{c}$ is a schematic illustration of FGDY-2.

Fig. 2a shows the Raman spectra of GDY and FGDYs with two distinct peaks, the $\mathrm{D}$ and $\mathrm{G}$ peaks. The D peak at approximately $1370 \mathrm{~cm}^{-1}$ indicates structural defects and the $G$ peak at approximately $1585 \mathrm{~cm}^{-1}$ was caused by the first-order scattering of the in-phase stretching vibration with the $\mathrm{E}_{2 \mathrm{~g}}$ mode. ${ }^{\mathbf{9 , 2 1}}$ The $I_{\mathrm{D} /} I_{\mathrm{G}}$ ratio increases from 0.77 for GDY to approximately 0.95 for FGDY-3, which means that the FGDYs had more defects that were caused by the F-doping. ${ }^{22}$ Furthermore, because of the vibration of the conjugated diyne-links $(-\mathrm{C} \equiv \mathrm{C}-\mathrm{C} \equiv \mathrm{C}-)$, two weak peaks at $1926 \mathrm{~cm}^{-1}$ and $2110 \mathrm{~cm}^{-1}$ for GDY have also appeared. ${ }^{9,22}$ However, these two characteristic peaks of FGDYs were weaker, which may be because of the incorporation of $\mathrm{F}$ atoms destroying the sp hybrid carbon atoms of GDY. ${ }^{23}$

Fig. 2b shows the survey XPS of the samples. The GDY displays three prominent peaks at $285.6 \mathrm{eV}, 532.1 \mathrm{eV}$ and $978.6 \mathrm{eV}$, corresponding to the photoemission of the $\mathrm{C} 1 \mathrm{~s}, \mathrm{O} 1 \mathrm{~s}$ and $\mathrm{O}$ Auger electron. ${ }^{22,24}$ The $\mathrm{O}$ atom was introduced by the adsorbed air in the synthesis process of GDY. For the FGDYs samples, the $\mathrm{F}$ 1s peak at $687 \mathrm{eV}$ and the $\mathrm{F}$ Auger peak at $832.5 \mathrm{eV}$ were seen clearly, ${ }^{22}$ and the $\mathrm{F}$ signals increased obviously with the increase of the mass ratios of GDY and $\mathrm{XeF}_{2}$ in the synthesis experiment. Fig. 2c gives the typical C 1s XPS spectra of GDY and FGDY. For GDY, four distinct peaks appeared at $284.5 \mathrm{eV}, 285.3 \mathrm{eV}, 287.2 \mathrm{eV}$ and $288.5 \mathrm{eV}$ in the $\mathrm{C} 1 \mathrm{~s}$ spectrum, which was attributed to the $\mathrm{C}-\mathrm{C}\left(\mathrm{sp}^{2}\right)$, the $\mathrm{C}-\mathrm{C}(\mathrm{sp})$, the $\mathrm{C}-\mathrm{O}$ and the $\mathrm{C}=\mathrm{O}$ bonds, respectively. ${ }^{9,19}$ The area ratio of $\mathrm{sp} / \mathrm{sp}^{2}$ was 2.5 , which indicated that the diacetyl bond had a better linkage to the benzene ring. ${ }^{9}$ In addition to the four peaks, FGDYs had two other peaks at $285.9 \mathrm{eV}$ and $290.7 \mathrm{eV}$, which corresponded to the $\mathrm{C}-\mathrm{F}$ bond and the $\mathrm{C}-\mathrm{F}_{2}$ bond, respectively. ${ }^{25,26}$ For FGDY-1, it was observed that the chemical 

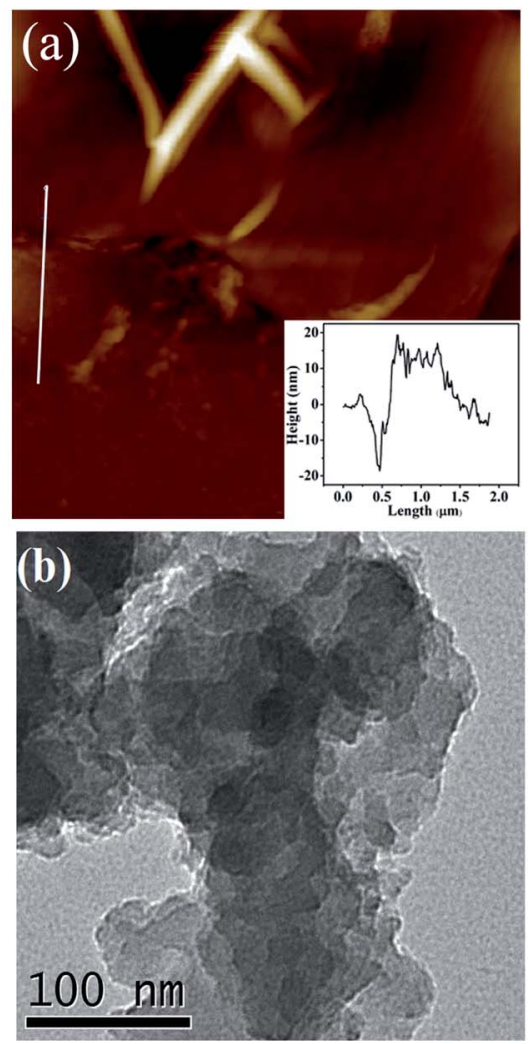

(c)

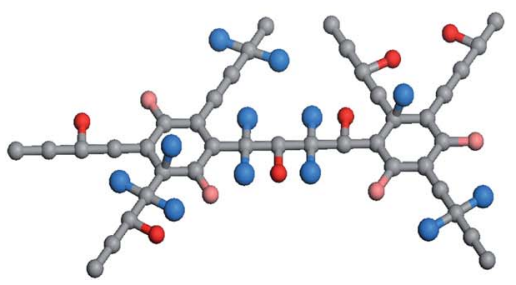

Fig. 1 (a) The AFM image and the lower right inset is the height profile, (b) the TEM image, (c) a schematic illustration of FGDY-2, in which gray balls represent $C$ atoms, blue balls represent $F$ atoms, red balls represent $\mathrm{O}$ atoms in the $\mathrm{C}-\mathrm{O}$ bonds and pink balls represent $\mathrm{O}$ atoms in $\mathrm{C}=\mathrm{O}$ bonds. structure of F only contained the $\mathrm{C}-\mathrm{F}$ bond. This was because the $\mathrm{F}$ atoms were preferentially doped at the triple bond of the chain and then doped on the ring in order to preserve the planar network structure of the GDY. ${ }^{27}$ Schematic illustrations of the F doping in GDY are presented in Fig. S1 (see ESI $\dagger$ ). The atomic percentages were obtained from the quantitative analysis of the XPS, as shown in Table 1. From FGDY-1 to FGDY-3, the proportion of the $\mathrm{C}-\mathrm{F}$ group was $0 \%, 16 \%$ and $19.7 \%$, respectively. The proportions of the $\mathrm{C}-\mathrm{F}_{2}$ group were $5.2 \%, 5 \%$ and $12.2 \%$, respectively. Meanwhile, the proportion of the sum of $\mathrm{C}-\mathrm{O}$ and $\mathrm{C}=\mathrm{O}$ groups increased from $19.5 \%$ with GDY to $39.8 \%$ with FGDY-3. This indicated that all the samples had oxygenrich groups. Meanwhile, the area ratio of $\mathrm{sp} / \mathrm{sp}^{2}$ from FGDY-1 to FGDY-3 was significantly reduced from 0.7 to 0.2 . This confirmed that the $\mathrm{F}$ atom had been successfully doped, resulting in the breaking of the alkyne bond between the two hexagons because the area ratio of $\mathrm{sp} / \mathrm{sp}^{2}$ reduced gradually. ${ }^{25}$

To investigate the fluorescence properties of the fluorinated GDY, the prepared samples were dissolved in ethanol $(0.1 \mathrm{mg}$ $\left.\mathrm{mL}^{-1}\right)$. For the samples which contained hydrophilic oxygen containing functional groups $(\mathrm{C}-\mathrm{O}$ and $\mathrm{C}=\mathrm{O})$, the prepared samples had good solubility in ethanol (Fig. S2, ESI $\dagger$ ). Fig. 3a shows the fluorescence spectra of FGDY-2 when their excitation wavelengths ranged from $260 \mathrm{~nm}$ to $480 \mathrm{~nm}$. This presents the strongest peak at approximately $480 \mathrm{~nm}$ when excited by $400 \mathrm{~nm}$ wavelength and showed an obvious excitation wavelength dependence, which can be commonly observed in other carbon materials such as graphene and fluorinated graphene. ${ }^{4,22}$ The colorful PL emission changed from blue to green as the excitation wavelength changed sequentially for FGDY-2. The fluorescence spectra of the other FGDYs and GDY are presented in Fig. S3 (see ESI $\dagger$ ). They all exhibited similar excitation wavelength dependent fluorescent properties. However, FGDY-1 and GDY emit colors from bright blue to deep purple and then back to bright blue, and then gradually changed to a green emission. In a similar way to graphene, GDY also had a small dielectric constant and a weak spin ratio coupling because it was mainly composed of light atoms such as $\mathrm{C}$ and $\mathrm{O}^{28}$ Therefore, it had strong carrier-carrier interactions and electronic states with well-defined spin multiplicities. Therefore, GDY, like the other two-dimensional carbon materials, had
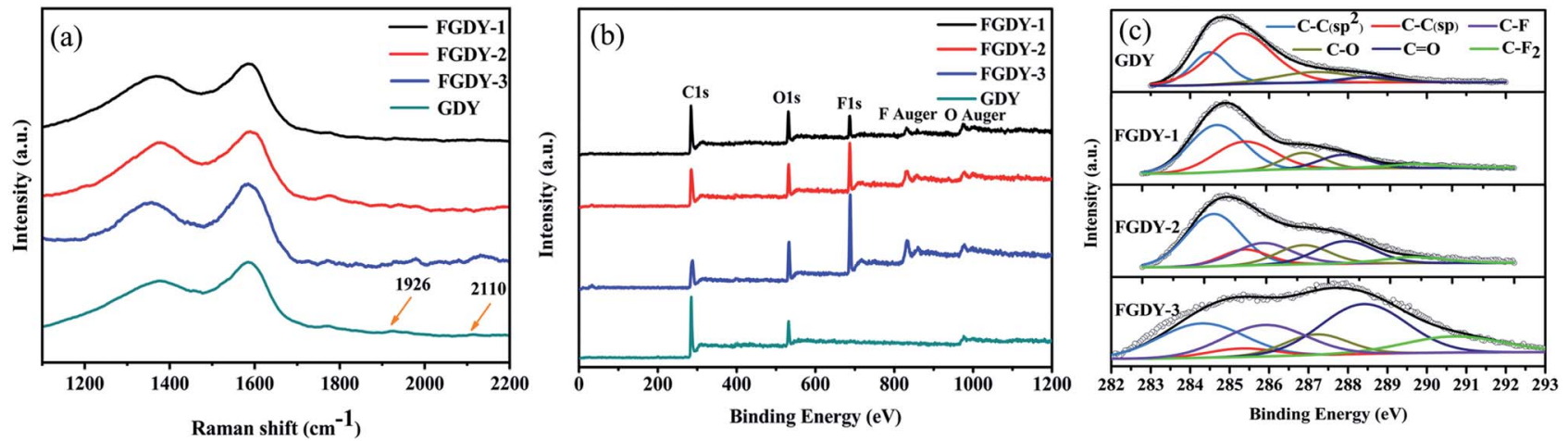

Fig. 2 (a) The Raman spectrum, (b) the survey XPS spectra of GDY and FGDYs, (c) high resolution XPS spectra of C 1s of GDY and FGDYs. 
Table 1 The composition of each element of GDY and FGDYs

\begin{tabular}{|c|c|c|c|c|c|}
\hline Chemical groups & $\mathrm{C}-\mathrm{C}\left(\mathrm{sp}^{2}\right)$ & C-C (sp) & $\mathrm{C}-\mathrm{O}, \mathrm{C}=\mathrm{O}$ & $\mathrm{C}-\mathrm{F}$ & $\mathrm{C}-\mathrm{F}_{2}$ \\
\hline Location (eV) & $284.5( \pm 0.2)$ & 285.3 & $287.2,288.5( \pm 0.1)$ & 285.9 & $290.7( \pm 0.1)$ \\
\hline FGDY-1 (\%) & 43 & 29.3 & 22.5 & 0 & 5.2 \\
\hline FGDY-3 (\%) & 23.7 & 4.6 & 39.8 & 19.7 & 12.2 \\
\hline GDY (\%) & 22.4 & 58.1 & 19.5 & 0 & 0 \\
\hline
\end{tabular}

a wider band structure than other conventional semiconductors of a similar size. ${ }^{28}$ At the same time, the presence of surface functional groups (such as carboxyl groups and hydroxyl groups) formed a few defect states, bringing discrete energy levels for GDY and FGDYs. ${ }^{28}$ Analogous to graphene, research has shown that the effective edge groups of green light were mainly carboxyl and amide groups, ${ }^{29}$ whereas hydroxyl groups contributed to the blue light emission. ${ }^{30}$ This also needs to be confirmed in future work on GDY and its derivatives.

Fig. 3b shows the fluorescence spectra of GDY and FGDYs at an excitation wavelength of $400 \mathrm{~nm}$. It was observed that the emission peak position of FGDYs was red-shifted gradually as the degree of fluorination increased. The FGDY-3 (the FGDY with the highest degree of fluorination) was red-shifted by approximately $20 \mathrm{~nm}$ compared to GDY. A higher degree of fluorination produced higher concentrations of surface defects that can capture more excitons. ${ }^{31}$ The generated excitons may be in some shallow trap states, resulting in a longer wavelength emission when the electron-hole pair recombines, and a redshift of the emission wavelength occurred. ${ }^{31}$

Compared with the pristine GDY, the fluorescence of FGDYs is significantly enhanced, and with the increase of the degree of fluorination, the fluorescence intensity presents an increasing trend. However, when the doping amount of fluorine atoms reaches $24 \%$, the fluorescence intensity decreased significantly. Therefore, the FGDY-2 (fluorine atom doping amount of $15.2 \%$ ) exhibited optimal blue emission properties. With rhodamine 6G as the standard reference material, the QE of FGDY-1, FGDY2, FGDY-3 were $1.7 \%, 3.7 \%$, and $1.6 \%$, respectively. The FGDYs showed a greatly improved QE compared to the original GDY of $0.7 \%$. According to density functional theory (DFT) calculations, the direct band gap of pristine GDY was from $0.44 \mathrm{eV}$ to $1.10 \mathrm{eV}$ when using the GW many-body theory. ${ }^{13}$ The value is corresponding to the PL which should be far away from bright blue to green colorful PL. Therefore, similar to GO, the oxygen-rich functional groups of GDY may be the source of the PL by
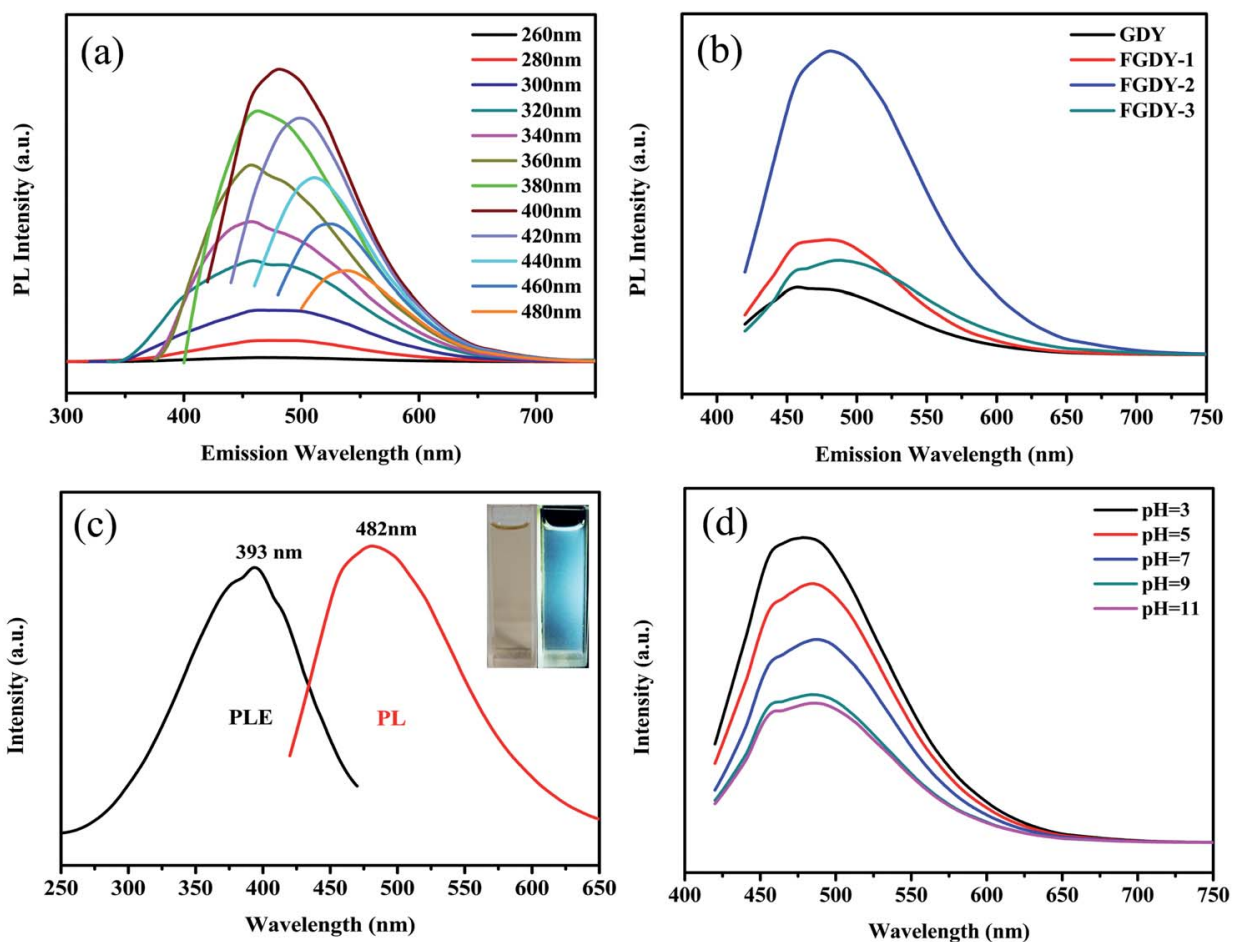

Fig. 3 (a) The PL spectra at different excitation wavelengths from $260 \mathrm{~nm}$ to $480 \mathrm{~nm}$ of FGDY-2, (b) the PL spectra at an excitation wavelength of $400 \mathrm{~nm}$ for all the samples, (c) the PLE spectrum with the detection wavelength of $482 \mathrm{~nm}$ (the black curve) and the PL spectra at an excitation wavelength of $400 \mathrm{~nm}$ (the red curve) of FGDY-2. In the inset, the image on the left is a solution of it dissolved in ethanol, and the image on the right is a solution irradiated with a $365 \mathrm{~nm}$ laser, (d) the PL intensity of FGDY-2 with different pH values at an excitation wavelength of $400 \mathrm{~nm}$. 
inducing the charge transfer between $\mathrm{C}$ and $\mathrm{O} .{ }^{\mathbf{2 1}}$ However, fluoride materials generally have low phonon energy $\left(<400 \mathrm{~cm}^{-1}\right),{ }^{32}$ which resulted in a low probability of nonradiative transitions, ${ }^{33}$ allowing free electrons in highest occupied molecular orbit (HOMO) to have more chances to return to the ground state, thus the luminescence obtained after doping with fluorine was significantly enhanced. This was also consistent with the results shown in Fig. 3a, where the greater the amount of F-doping, the smaller the fluorescent colourful emission range of FGDY was. However, the $\mathrm{F}$ atom reduces the HOMO and the lowest unoccupied molecular orbit (LUMO) energy levels and enhanced the migration of charge carriers. ${ }^{34}$ Therefore, compared with the pristine GDY, the PL intensity of FGDYs was greatly enhanced. When the doping amount of fluorine and oxygen atoms were excessive, the probability of transition of the free electron to the ground state was reduced, resulting in fluorescence quenching because of the energy transfer between the oxygen-rich functional group and the $\mathrm{F}$ atoms. ${ }^{35}$ Thus, the fluorescence intensity of FGDY-3 with high fluorine and oxygen doping amounts was remarkably weakened.

Fig. 3c shows PL and photoluminescence excitation (PLE) spectrum of FGDY-2. It was easily seen that the strongest blue emission was at $482 \mathrm{~nm}$ when excited by the $400 \mathrm{~nm}$ wavelength. Meanwhile, the PLE spectra under the $482 \mathrm{~nm}$ showed strong peaks at about $393 \mathrm{~nm}$. The inset of Fig. 3c shows that the FGDY-2 solution emitted an obvious blue luminescence. The PL intensity of FGDY-2 at different $\mathrm{pH}$ values is shown in Fig. 3d. The $\mathrm{pH}$ is an important parameter in many engineering experiments (such as biotechnology, environmental monitoring, and so on), and being able to easily monitor is also important. The fluorescence intensity of FGDY has an interesting change under different $\mathrm{pH}$ conditions at room temperatures. Unlike the PL of other carbon materials which quenched gradually under acidic conditions, ${ }^{31}$ the FGDYs showed an increase in PL intensity as the acidity of the solution increased. This might be because of the surface-doped $\mathrm{F}$ atoms, dramatically affecting the surface energy of the F-containing carbon materials which acted as a protective shell with a mechanism similar to that of surface covered amino groups in silicon QDs. ${ }^{31,36,37}$ At the same time, it was seen that the intensity of PL continued to decrease under alkaline conditions. The decrease of PL intensity can be induced by the PL center destruction caused by the hydroxy groups. ${ }^{38,39}$ As shown in Fig. S4 (see ESI $\dagger$ ), the peak of the surface adsorbed hydroxyl groups (approximately $3400 \mathrm{~cm}^{-1}$ ) was clearly seen at alkaline conditions. ${ }^{40}$ This indicated that in an alkaline environment, the adsorbed hydroxyl groups of FGDY may distinctly affect the intensity of PL. The sensitivity of PL properties to $\mathrm{pH}$ conditions might be useful for new ideas for the application of carbon-based materials in the field of environmental detection.

\section{Conclusions}

In summary, an efficient and convenient method for synthesizing fluorinated GDY was proposed by heating a mixture of XeF2 and GDY. From the XPS analysis, it is confirmed that the pristine GDY has a non-negligible oxygen content, and the degree of fluorination is varied by using different mass ratios of $\mathrm{XeF}_{2}$ and GDY. The $\mathrm{F}$ atoms are preferentially doped at the triple bond of the chain. From the PL spectra, it is found that the fluorescence of GDY is enhanced because of the fluorine doping. It was observed that FGDY-2 (with a fluorine atom doping amount of $15.2 \%$ ) has the strongest blue emission at $482 \mathrm{~nm}$. The enhancement of PL is because of a low probability of non-radiative transitions in fluoride materials. In addition, as the $\mathrm{pH}$ value increases from 3 to 11 , the fluorescence intensity of FGDY-2 gradually decreases, showing an environmental sensitivity. The FGDYs have a great future potential in biology, optoelectronics and environment detection because of their tunable optical properties and low cytotoxicity.

\section{Conflicts of interest}

There are no conflicts to declare.

\section{Acknowledgements}

We acknowledge the financial support by the Natural Science Foundation of China (No. 11404059), and the Natural Science Foundation of Fujian Province (Grant No. 2017J01035, No. 2016J01010).

\section{Notes and references}

1 W. Qing Hua, K. Z. Kourosh, K. Andras, J. N. Coleman and M. S. Strano, Nat. Nanotechnol., 2012, 7, 699-712.

2 Y. Je Min, K. J. Hyoun and H. Byung Hee, Chem. Soc. Rev., 2015, 44, 4835-4852.

3 E. Morales-Narváez and A. Merkoçi, Adv. Mater., 2012, 24, 3298-3308.

4 Q. Mei, K. Zhang, G. Guan, B. Liu, S. Wang and Z. Zhang, Chem. Commun., 2010, 46, 7319-7321.

5 J. Shen, Y. Zhu, X. Yang, J. Zong, J. Zhang and C. Li, New J. Chem., 2012, 36, 97-101.

6 W. Andrew Leigh, S. Hasan, J. Ki-Joon, B. Aaron, H. Seyda, K. Roland, S. Florian, O. Markus, N. Peter and M. Michael, ACS Nano, 2014, 8, 7801-7808.

7 J. Ki-Joon, L. Zonghoon, P. Elad, M. Luca, B. Aaron, P. CheolMin, M. Rueben, R. Velimir, K. Robert and T. J. Richardson, ACS Nano, 2011, 5, 1042-1046.

8 F. a. M. N. Noor, S. B. M. Ashraf, S. M. Mat and U. A. Ali, Opt. Mater., 2018, 83, 306-314.

9 G. Li, Y. Li, H. Liu, Y. Guo, Y. Li and D. Zhu, Chem. Commun., 2010, 46, 3256-3258.

10 C. Huang, Y. Li, N. Wang, Y. Xue, Z. Zuo, H. Liu and Y. Li, Chem. Rev., 2018, 118, 7744-7803.

11 Z. Jia, Y. Li, Z. Zuo, H. Liu, C. Huang and Y. Li, Acc. Chem. Res., 2017, 50, 2470-2478.

12 J. J. Zheng, Z. Xiang, Y. Zhao and X. Gao, Sci. Rep., 2013, 3, 1271.

13 G. Luo, X. Qian, H. Liu, R. Qin, J. Zhou, L. Li, Z. Gao, E. Wang, W.-N. Mei, J. Lu, Y. Li and S. Nagase, Phys. Rev. $B, 2011,84,075439$. 
14 H. Yu, Y. Xue, L. Hui, C. Zhang, Y. Li, Z. Zuo, Y. Zhao, Z. Li and Y. Li, Adv. Mater., 2018, 30, 1707082.

15 S. Thangavel, K. Krishnamoorthy, V. Krishnaswamy, N. Raju, S. J. Kim and G. Venugopal, J. Phys. Chem. C, 2015, 119, 22057-22065.

16 Z. Jin, Q. Zhou, Y. Chen, P. Mao, H. Li, H. Liu, J. Wang and Y. Li, Adv. Mater., 2016, 28, 3697-3702.

17 B. Bhattacharya, N. B. Singh and U. Sarkar, Int. J. Quantum Chem., 2015, 115, 820-829.

18 J. Koo, H. J. Hwang, H. Bing, H. Lee, H. Lee, Y. Kwon, S. H. Wei and H. Lee, J. Phys. Chem. C, 2013, 117, 1196011967.

19 S. Zhang, H. Liu, C. Huang, G. Cui and Y. Li, Chem. Commun., 2015, 51, 1834-1837.

20 J. Koo, M. Park, S. Hwang, B. Huang, B. Jang, Y. Kwon and H. Lee, Phys. Chem. Chem. Phys., 2014, 16, 8935-8939.

21 Z. Yong-Ping, F. Qian, T. Nu-Jiang and D. U. You-Wei, New Res. Carbon Mater., 2018, 33, 516-521.

22 Q. Feng, W. Xiao, Y. Liu, Y. Zheng, Y. Lin, J. Li, Q. Ye and Z. Huang, Materials, 2018, 11, 91.

23 S. Zhang, H. Du, J. He, C. Huang, H. Liu, G. Cui and Y. Li, ACS Appl. Mater. Interfaces, 2016, 8, 8467-8473.

24 M. Bruna, B. Massessi, C. Cassiago, A. Battiato, E. Vittone, G. Speranza and S. Borini, J. Mater. Chem., 2011, 21, 18730-18737.

25 S. Zhang, Y. Cai, H. He, Y. Zhang, R. Liu, H. Cao, M. Wang, J. Liu, G. Zhang, Y. Li, H. Liu and B. Li, J. Mater. Chem. A, 2016, 4, 4738-4744.

26 G. J. Ross, J. F. Watts, M. P. Hill and P. Morrissey, Polymer, 2000, 41, 1685-1696.
27 Y. Li, L. Xu, H. Liu and Y. Li, Chem. Soc. Rev., 2014, 43, 2572. 28 S. Zhu, Y. Song, J. Wang, H. Wan, Y. Zhang, Y. Ning and B. Yang, Nano Today, 2017, 13, 10-14.

29 S. Zhu, J. Shao, Y. Song, X. Zhao, J. Du, L. Wang, H. Wang, K. Zhang, J. Zhang and B. Yang, Nanoscale, 2015, 7, 79277933.

30 W. Lei, Z. Shou-Jun, W. Hai-Yu, Q. Song-Nan, Z. Yong-Lai, Z. Jun-Hu, C. Qi-Dai, X. Huai-Liang, H. Wei and Y. Bai, ACS Nano, 2014, 8, 2541-2547.

31 H. Sun, H. Ji, E. Ju, Y. Guan, J. Ren and X. Qu, Chemistry, 2015, 21, 3791-3797.

32 F. Wang and X. Liu, Chem. Soc. Rev., 2009, 38, 976-989.

33 F. Vetrone and J. A. Capobianco, Int. J. Nanotechnol., 2008, 5, 1306-1339.

34 F. Houshmanda, S. Jalili and J. Schofield, Phys. Chem. Res., 2016, 4, 231-243.

35 B. Zheng, S. Xu, L. Lin, Z. Wang, Z. Feng and Z. Zheng, Opt. Lett., 2015, 40, 2630-2633.

36 Y. L. Zhong, F. Peng, F. Bao, S. Y. Wang, X. Y. Ji, L. Yang, Y. Y. Su, S. T. Lee and Y. He, J. Am. Chem. Soc., 2013, 135, 8350-8356.

37 P. Gong, Z. Yang, W. Hong, Z. Wang, K. Hou, J. Wang and S. Yang, Carbon, 2015, 83, 152-161.

38 P. R. Ehrmann, K. Carlson, J. H. Campbell, C. A. Click and R. K. Brow, J. Non-Cryst. Solids, 2004, 349, 105-114.

39 S. Yubin, Z. Shoujun, X. Siyuan, Z. Xiaohuan, Z. Junhu, Z. Hao, F. Yu and Y. Bai, Nanoscale, 2014, 6, 4676-4682.

40 M. R. Su, Z. X. Wang, H. J. Guo, X. H. Li, S. L. Huang, W. Xiao and L. Gan, Electrochim. Acta, 2014, 116, 230-236. 